\title{
Generation of Synthetic Multifractal Realistic Surfaces Based on Natural Model and Lognormal Cascade: Application to MRI Classification
}

\author{
Mohamed Khider ${ }^{1}$, Abdelmalik Taleb-Ahmed ${ }^{2}$, and Boualem Haddad ${ }^{1}$ \\ 1 Université des Sciences et de la Technologie Houari Boumedienne, LTIR, Algeria \\ 2 Université de Valenciennes et du Hainaut Cambrésis, LAMIH, FRE 3304, France \\ taleb@univ-valenciennes.fr
}

\begin{abstract}
This paper presents a method of generating realistic synthetic multi-fractals surfaces, constructed with multiplicative cascades, that follow lognormal probability density function. The conservation of the natural image gradient direction, and the variance of the difference minimization at each scale between natural image multipliers and those of the selected lognormal model, preserves the initial texture structure. Validation of the model is made with wavelet leader based multifractal analysis, we also propose an application to MRI classification of trabecular bone texture, to differentiate between healthy and osteoporotic cases.
\end{abstract}

Keywords: lognormal cascade, Discrete Wavelet Transform, wavelet leader, multifractal analysis, Monte-Carlo sampling, Iterative Conditional Modes (ICM), Markov Random Fields (MRF), probabilistic model and Bayesian classification.

\section{Introduction}

Over the past few decades, an important number of texture classification approaches have been explored, and numerous applications relating to computer vision and pattern recognition have been found, including industrial, remote sensing and medical imaging. These approaches can be divided into four categories: statistical, structural methods, modelbased, and signal processing methods [1. In this work, the classification is performed in two steps : 1. firstely, we begin by generating the realistic multifractal surfaces from our serie of image, with a fixed predominant Holder exponent, 2. secondly, we calculate the percentage of correspondence between the original image and their multifractal version. Therefore, in our application of MRI trabecular bone texture classification, we begin by generating the multifractal version of the ROI (region of interest) as explained in section two, the validation of the model is performed by using wavelet leader based multifractal analysis in section three.To generate several prototypes from the multifractal version of our image, Markov random 
fields are used as shown in section four. In this paper, detection of similarity is performed by Bayesian classification and comparison of coincidence between valley's pixel from trabecular texture is presented in section four.

\section{Generation of the Natural Multifractal Model}

Kolmogorov and Obukhov 23] have proposed the lognormal model that reveals multifractal behavior, afterward created from a $\mathcal{W}$ random multiplicative cascade process in Yalgom's work to generate intermittent [456]. Parallel to that, in the turbulence analysis, since the first works of Obukhov [7], the decomposition of signals into high and low-frequency parts did not cease to find favorable ground. Advent of Daubechies wavelet with compact support and fast algorithm implementation, on dyadic scales has made conceivable the synthesis of multifractal surfaces based on lognormal and log-poisson cascades in the relevant Arneodo et al articles of 2D-WTMM multifractal analysis [8]. We start by choosing the model, for example a Brodatz's texture, the product of convolution between the $f_{m}(x, y)$ model and the $\psi_{j, k, l}^{m}$ wavelet give us the coefficients according to the $\mathrm{m}$ direction, $(j, k)$ position and the scale of analysis $l$ :

$$
C_{j, k, l}^{m}=\left\langle f_{m}(\mathrm{x}, \mathrm{y}), \psi_{j, k, l}^{m}\right\rangle
$$

The 2D-DWT modulus are expressed by :

$$
d_{j, k, l}=\sqrt{\sum_{m=1}^{3}\left[C_{j, k, l}^{m}\right]^{2}}
$$

The directions of the real model $f_{m}(x, y)$ coefficients must be preserved, hence the angles $\varphi$ and $\theta$ are estimated by using the arguments :

$$
\begin{gathered}
\varphi_{j, k, l}=\arg \left(\sqrt{\sum_{m=1}^{2}\left[C_{j, k, l}^{m}\right]^{2}}+i C_{j, k, l}^{2}\right) \\
\theta_{j, k, l}=\arg \left(C_{j, k, l}^{1}+i C_{j, k, l}^{2}\right)
\end{gathered}
$$

Let $w_{l}$ be the multipliers at scale $l$ that follow a lognormal law, by minimizing the variance of difference between $w_{m}$ multipliers and those generated randomly $w_{i}$ following a lognormal probability density function, this calculation is repeated many times until stability of the result :

$$
\begin{aligned}
d_{j, k, l} & =d_{j, k, l-1} w_{m-1} \\
\operatorname{var}\left(w_{m}-w_{l}\right) & =\min _{w_{i}}\left[\operatorname{var}\left(w_{m}-w_{i}\right)\right]
\end{aligned}
$$

Then, we proceed as in Decoster et all works [9], with the iteration from the coarser to the finer scale :

$$
M_{j, k, l}=M_{j, k, l-1} w_{l-1}
$$


The wavelet coefficients of synthetic surface $G_{j, k, l}$ are written according to the $M_{j, k, l}$ modules through :

$$
\begin{gathered}
G_{j, k, l}^{1}=\cos \left(\varphi_{j, k, l}\right) \cos \left(\theta_{j, k, l}\right) M_{j, k, l} \\
G_{j, k, l}^{2}=\cos \left(\varphi_{j, k, l}\right) \sin \left(\theta_{j, k, l}\right) M_{j, k, l} \\
G_{j, k, l}^{3}=\sin \left(\theta_{j, k, l}\right) M_{j, k, l}
\end{gathered}
$$

The construction of the synthetic surface $f_{s}(\mathrm{x}, \mathrm{y})$ is performed with the following addition ( $\mathrm{N}$ is the total number of scale) :

$$
f_{s}(\mathrm{x}, \mathrm{y})=\sum_{j=0}^{+\infty} \sum_{k=0}^{+\infty} \sum_{l=0}^{N} \sum_{m=1}^{3} G_{j, k, l}^{m} \psi_{j, k, l}^{m}(\mathrm{x}, \mathrm{y})
$$

Such in the case of our surfaces $f_{s}(\mathrm{x}, \mathrm{y})$, a generated surface by lognormal cascade is characterized with the multifractal spectrum :

$$
D(h)=-\frac{(h+\mu / \ln (2))}{2 \sigma^{2} / \ln (2)}+2
$$

When $\mu$ and $\sigma^{2}$ denote respectively the mean and the variance from the $w_{l}$ lognormal law.

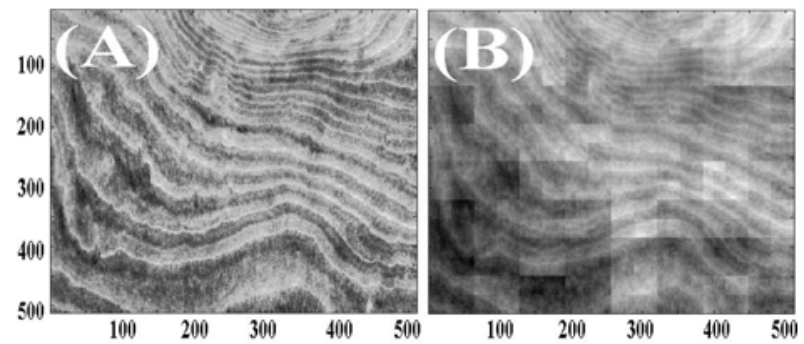

Fig. 1. (A) Brodatz's texture, and in (B) its multifractal version $h(q=0)=0.38$, $\mu=-0.38 \log (2)$ and $\sigma^{2}=0.03 \log (2) \operatorname{lognormal}$ cascade parameter obtained by the first order Daubechies wavelet

\section{Validation of the Model}

To estimate the multifractal spectrum, the leading coefficients method of S. Jaffard [10] is used, and we employ the same notation as B. Lashermes [1]. In the case of one dimension for example, $d_{f}(j, k)$ indicate the discrete wavelet transform coefficient for each dyadic interval in scale $2^{j}$, then $l_{f}(j, k)$ the leading coefficient is obtained by the relation :

$$
l_{f}(j, k)=\sup _{\lambda^{\prime} \subset 3 \lambda(j, k), j^{\prime} \leqslant j}\left|d_{f}\left(\lambda^{\prime}\right)\right|
$$


The structure function is given by the following equation $(d=2)$ :

$$
S_{f}^{l}(q, j)=\frac{1}{n(j)} \sum_{\left\{k_{i}\right\}} \frac{1}{2^{d}-1} \sum_{m}\left(l_{f}\left(j, k_{i}, m\right)\right)^{q}
$$

If we note, by $q$ the moment and $n(j)$ the number of leading coefficients at the octave $j$, the scale function is specified by $\zeta_{l}^{f}(q)$ and satisfies the relation :

$$
S_{f}^{l}(q, j) \sim 2^{j \zeta_{f}^{l}(q)}
$$

The Legendre spectrum $D(h)$ is given by the multifractal formalism :

$$
D_{f}^{l}(h)=\min _{q}\left(d+q h-\zeta_{f}^{l}(q)\right)
$$
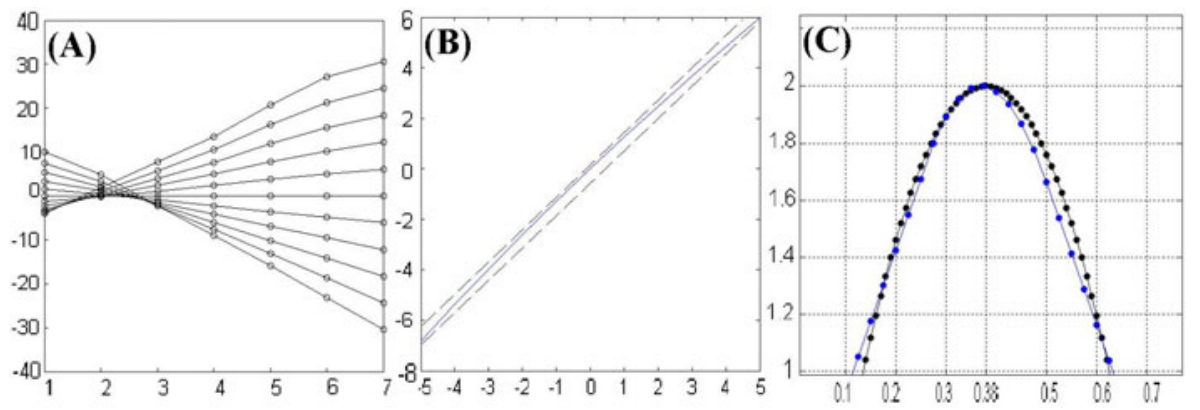

Fig. 2. Multfractal analysis of image (1.B) in (A) Partition functions $-5 \leq q \leq+5$ with axis $(x, y)=\left(j, S_{f}^{l}(q, j)\right)$, (B) Scale function with axis $(x, y)=\left(q, \zeta_{l}^{f}(q)\right)$, and in (C) The Legendre spectrum with multifractal behavior and axis $(x, y)=\left(h, D_{f}^{l}(h)\right)$, wavelet leader based multifractal analysis is performed by second order Daubechies wavelet, in black color the theoretical and in blue the experimental spectrum

\section{Application: MRI Classification}

We present a method of bone texture classification based on Monte-Carlo sampling, following a uniform probability distribution. A great similarity is observed in the texture structure between the original image and the multifractal model when we generate a multifractal model with the most frequent Holder exponent close to the exponent of the original image. In addition, we have used Markov's chains for restoration. In the beginning Markov has developed his theory to analyze the Pushkin's literary text [12], then the appearance of Markov random fields is due to statistical physics in the work of Ising, and its theoretical basis were described by Preston and Spitzer [13]. 


\subsection{The Proposed Method}

Trabecular structures pixels and valleys pixels are detected by Bayesian classification, this requires a learning step to define the prior probability of gray levels (according to the mode of acquisition, one image is enough for a whole series). Next, the estimation of coincidence between pixels of the same nature is performed. Furthermore, we propose the combination of Markov random fields (MRF) restoration method of second order and the iterative conditional modes (ICM) with 6 cycles and $\beta=1.5$, this allow the production of several prototypes of our multifractal model, and increases at lower cost (in number of operations) the statistical population. The preliminary results are very encouraging and showing $100 \%$ of good classification on 10 MRI images (Table.1), and a dramatically reduced in computing time by using Monte Carlo sampling is observed. The algorithm can be implemented in parallel (multi processing). To generate the prototype, ICM and MRF are used, we proceed as follows : for any scene $x^{*}$ which represents the central pixel obtained by Monte Carlo sampling and the second order MRF neighbors, We calculate the mean $\mu\left(x_{i}^{*}\right)$ and the variance $\sigma^{2}$, and generate the $y_{i}$ variable from Gaussian probability distribution function with the same mean and variance as $x^{*}$ scene. The $y_{i}$ variable represents the generated gray level. The probability that the $k$ gray level is associated with pixel at the position $i$ is such that [14] :

$$
P_{i}(k / .)=e^{\beta u_{i}(k)} / \sum_{l=1}^{c} e^{\beta u_{i}(l)}
$$

The variable $u_{i}(l)$ indicate the number of $i$ neighbors possessing $l$ gray level, $\beta$ is a fixed parameter with a 1.5 value and 6 ICM cycles such as in Besag et al article. The ICM corresponds to a succession of successful expression reduction (improving) :

$$
\frac{1}{2 \sigma^{2}}\left\{y_{i}-\mu\left(x_{i}\right)\right\}^{2}-\beta u_{i}^{*}\left(x_{i}\right)
$$

With $u_{i}^{*}\left(x_{i}\right)$ which gives the current number of $i$ neighbors that have the same gray level as the pixel $i$. To estimate the correspondence of pixels (trabecular structures, valleys), we use a probabilistic model and a Bayesian classification, $y$ indicates the gray level, $T$ and $V$ illustrate respectively trabecular structures and valleys pixels. The conditional a posteriori probabilities are obtained by [15]:

$$
\begin{aligned}
& P(T / y)=\frac{P(T) P(y / T)}{P(T) P(y / T)+P(V) P(y / V)} \\
& P(V / y)=\frac{P(V) P(y / V)}{P(T) P(y / T)+P(V) P(y / V)}
\end{aligned}
$$

The a priori probabilities $P(T), P(V), P(y / T)$ and $P(y / V)$ are estimated with a learning step performed by an expert (a doctor indicates the trabecular structures and valleys regions for best results, to do this, we suggest a pathological 


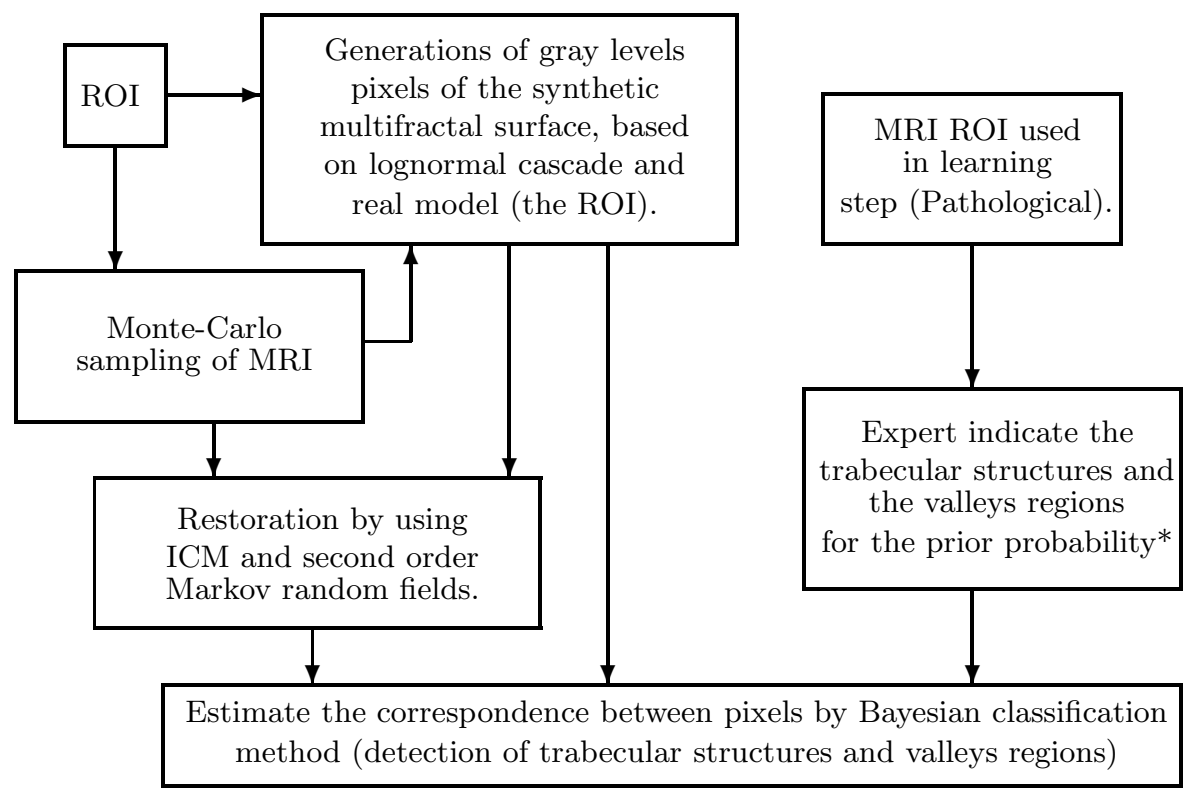

Fig. 3. Block diagram of the method, and the principal steps to follow. (*) this step is needed for training to estimate the prior probabilities.

ROI (fig.4)), a trabecular structure pixel is detected if $P(T / y)>\alpha P(V / y)$, in our case, we took $\alpha=1$, it can be modified for example to reduce the risk of considering a valley pixel as a trabecular structure pixel, we must choose $0<\alpha<1$.

\subsection{Results and Discussion}

In our application, the method of Monte Carlo is employed with $10^{4}$ samples, and the first order Daubechies wavelets are used during the reconstruction step with lognormal cascade multipliers, it requires a range of 8 octaves, for a ROI of $512 \times 512$ pixels. Ten MRI images are used, the choice of the most frequent exponent is performed with $h(q=0)=0.1$ value that best reflects the pathological ROI structure, indeed, the rate of coincidence of pixels in the regions of same nature (trabecular structure or valleys) is higher than in the case of trabecular texture of normal patient (Table.1). If we consider the totality of the ROI, using the ICM restoration method improve the homogeneity of the texture by reducing artifacts (due to the DWT dyadic grid)(fig.5 and fig.6). We can generate several prototypes from the same image (the table shows the result after one operation of restoration). We can distinguish unambiguously the two case of studies, furthermore, using MRF allowed us to increase the difference between average of the two classes. We should note, that the a priori probability is obtained after a training step by using a pathological ROI, since in this case, it's easier to distinguish between the two regions trabecular structures and valleys. 
Table 1. The pathological ROI cases and its results are represented in bold characters, we give the percentage of coincidence between trabecular structures pixels and valleys pixels detected by Bayesian classification. MF indicates that the comparison is made between the ROI and its multifractal version. MF ICM indicates that the comparison is made between the ROI and its multifractal version restored by ICM method.

\begin{tabular}{lllllllllll}
\hline ROI & B & C & E & G & K & M & O & S & T & U \\
\hline MF & 19.24 & $\mathbf{4 5 . 7 8}$ & $\mathbf{4 5 . 7 4}$ & 26.21 & $\mathbf{4 4 . 9 4}$ & $\mathbf{4 2 . 6 4}$ & 26.23 & 28.72 & 22.13 & $\mathbf{4 3 . 7}$ \\
MF ICM & 18.6 & $\mathbf{4 2 . 5 5}$ & $\mathbf{4 4 . 1}$ & 26.49 & $\mathbf{4 1 . 1 9}$ & $\mathbf{3 9 . 0 1}$ & 22.26 & 22.39 & 18.23 & $\mathbf{4 2 . 3 2}$ \\
\hline
\end{tabular}
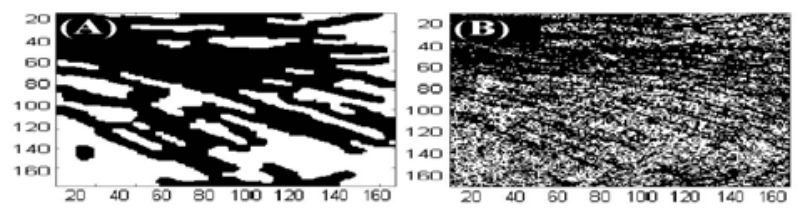

Fig. 4. (A) Training step, in black color the trabecular structures pixels region, the first figure from left illustrate the regions chosen by an expert (to estimate the a priori probabilities), and (B) indicate the detected regions by Bayesian classification of the same pathological ROI (the a posteriori probabilities)
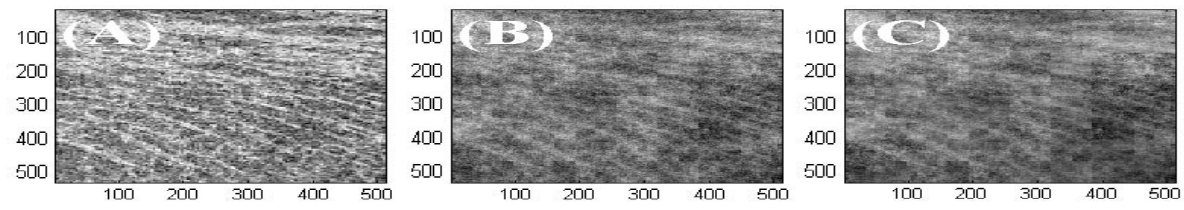

Fig. 5. (A) pathological MRI ROI, (B) its multifractal version $h(q=0)=0.1, \mu=$ $-0.1 \log (2)$ and $\sigma^{2}=0.01 \log (2)$, and in $(\mathbf{C})$ the same multifractal version restored with ICM
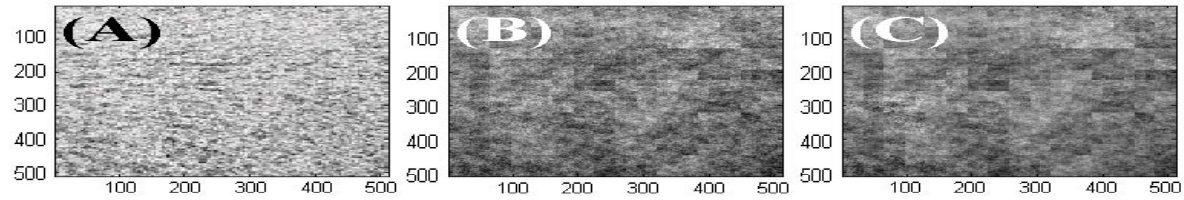

Fig. 6. (A) healthy MRI ROI, (B) its multifractal version $h(q=0)=0.1, \mu=$ $-0.1 \log (2)$ and $\sigma^{2}=0.01 \log (2)$, and in (C) the same multifractal version restored with ICM 


\section{Conclusions}

This paper concerns the use of multifractal surfaces generation method based on the extraction of attributes from natural images such as cascade multipliers and the gradients directions from the wavelet of decomposition, what makes the synthetic image more realistic. An application of texture classification is derived, an osteoporotic bone texture and a healthy one are compared with their multifractals versions resulting from lognormal cascade with a fixed predominant Holder exponent, a greater similarity is observed between original texture and that generated with lognormal cascade when the Holder exponents are close together (pathological case in our application). Preliminary results are very promising and as perspective an automatic method is envisaged and it would be interesting to apply to other types of textures for classification or segmentation.

\section{References}

1. Bharati, M.H., Liu, J.J., MacGregor, J.F.: Image texture analysis: methods and comparisons. J. Chemometrics and Intelligent Laboratory Systems 72, 57-71 (2004)

2. Kolmogorov, A.N.: A refinement of previous hypotheses concerning the local structure of turbulence. J. Fluid Mech. 13, 83-85 (1962)

3. Obukhov, A.M.: Some specific features of atmospheric turbulence. J. Fluid Mech. 13, 77-81 (1962)

4. Bacry, E., Muzy, J.F.: Log-infinitely divisible multifractal processes. Comm. In Math. Phys. 236, 449-475 (2003)

5. Chainais, P., Li, J.-J.: Synthése de champs scalaires multifractals: application à la synthése de texture. In: GRETSI 2005, Louvain-la-Neuve, Belgique (2005)

6. Schmitt, F.G.: Intermittence et turbulences; analyse de données, validation de modéles et applications, HDR, Université Paris 6 (2001)

7. Frisch, U.: Turbulence. The legacy of A.N. Kolmogorov. Cambridge University Press, Cambridge (1995)

8. Arneodo, A., Decoster, N., Roux, S.G.: A wavelet-based method for multifractal image analysis. I. Methodology and test applications on isotropic random rough surface. European Physical Journal B 15, 567-600 (2000)

9. Arneodo, A., Decoster, N., Roux, S.G.: A wavelet-based method for multifractal image analysis. II. Applications to synthetic multifractal rough surface. European Physical Journal B 15, 739-764 (2000)

10. Jaffard, S.: Multifractal formalism for function, part 1: Results valid for all functions. S.I.A.M: Journal of Mathematical Analysis 28, 944-970 (1997)

11. Lashermes, Analyse multifractale pratique: coefficients dominants et ordres critiques. applications à la turbulence pleinement développé. effets de nombre de reynolds fini, Ph.D. thesis (2005)

12. Duda, R.O., Hart, P.E., Stork, D.G.: Pattern Classification. Edition Wiley InterScience, New York (2001)

13. Kindermann, R., Laurie Snell, J.: Markov Random Fields and their applications. American Mathematical Society, Providence (1980)

14. Besag, J.: On the statistical analysis of dirty pictures. Journal of Royal Statistical Society. Series B (Methodological) 48, 259-302 (1986)

15. Pieczynski, W.: Modèles de Markov en traitements d'images. Traitement Du Signal 20, 255-278 (2003) 